\title{
Historic specimens collected from the Korean Peninsula in the early 20th century (II)
}

\author{
Eun-Mi SUN, Kae Sun CHANG, Hyun-Duk SON ${ }^{1}$ and Hyoung-Tak IM* \\ Division of Forest Biodiversity, Korea National Arboretum of the Korea Forest Service, Pocheon 11186, Korea \\ ${ }^{1}$ Mokpo Natural History Museum, Mokpo 58699, Korea \\ ${ }^{2}$ Department of Biology, Chonnam National University, Gwangju 61186, Korea
}

(Received 11 June 2019; Revised 22 September 2019; Accepted 27 September 2019)

\begin{abstract}
Many of the historic plant specimens collected on the Korean Peninsula in the early twentieth century were lost during the Korean War, though some of them were deposited in the herbarium of Tokyo University (TI) and thus remained unharmed. Data on historic Korean plant specimens at TI are very important given the lack of current data on North Korean plants. Moreover a number of unidentified Korean historic specimens are present at TI. We carried out an identification process and created a list of plants in a newly found collection held by Dr. Ikuma Yoichiro, a Japanese entomologist. He traveled from Cheongjin to Hyesanjin via Baekdu-san (Mt.) in August of 1913 and collected 240 species. We also secured one duplicate set.
\end{abstract}

Keywords: The historic plant specimen, early 20th century, Korean Peninsula, TI, Ikuma Y., rare plants

한반도 자생식물이 근대적 방법으로 채집되어 서구 식 물학계에 알려진 것은 19 세기 중엽 이후의 일이다. 1854 년 프러시아 해군 제독 Alexander Schlippenbach는 한반도 동해안을 측량하는 과정에서 채집한 식물을 네덜란드의 F. A. W. Miquel에게 보냈으며, 이 표본들에 기초하여 버드 나무(Salix koreensis Anderss.)와 철쭉(Rhododendron schlippenbachii Maxim.)이 기재되었다. 이후 영국의 R. Oldham과 A. W. Carles, 일본의 Y. Hanabusa와 같은 비전문 가들의 채집이 있었고, 이들의 이름은 대나물(Gypsophila oldhamiana Miq.), 분꽃나무(Viburnum carlesii Hemsl.), 금 강초롱(Hanabusaya asiatica Nakai)의 학명에 담겨 있다. 20세기에 들어서면서 프랑스 선교사인 R. P. U. Faurie (쥐 오줌풀, Valeriana fauriei Briq.), E. J. Taquet (해변취, Saussurea taquetii Lev. \& Vant.), 일본의 T. Uchiyama (그늘 취, Saussurea uchiyamana Nakai)에 의해 식물채집을 목적 으로 하는 본격적인 활동이 시작되며, T. Nakai로 대표되 는 일본 학자들에 의한 식물분류학 연구가 이루어지게 된다.

19 세기 중엽에서 20 세기 중엽에 걸친 100 년 동안의 채 집품과 그에 근거한 종 인식은 한반도 근대 식물 연구의 기초가 되었으며, 이들 분류군에 대한 현대적 검증은 지 금 우리의 몫으로 남겨져 있다. 이 같은 인식에서 초기 연
구자들의 채집 행적 및 채집품은 중요한 기초자료가 되 며, R. P. U. Faurie (Chang et al., 2004), T. Nakai (Kim et al., 2006), T. Uchiyama (Kim et al., 2007), T. Ishidoya (Chang and Chang, 2010), T. Mori (Kim et al., 2012), G. Koidzumi, J. Ohwi, S. Kitamura (Chang et al., 2013) 등의 채집 일정에 대한 상세한 연구가 이루어졌다. 이러한 연구들은 주로 문헌에 인용된 표본 및 표본관(TI와 $\mathrm{KYO})$ 소장 표본의 라벨 정보에 근거한다. 강원대 이우철 교수는 1994년 연 구년 동안 TI 소장 한반도 표본의 이미지 및 라벨을 조사 하였다. 이상의 선행 연구로 축적된 한반도 고표본 기초 정보 $\mathrm{DB}$ 는 약 30,000 건에 이른다. Im et al. (2016)은 도쿄 대학 표본관에 미동정 상태로 보관되어 있는 한반도 고 표본을 조사하여 기존의 고표본 $\mathrm{DB}$ 에 추가하였다. 본 연 구는 도쿄대학 소장 미동정 한반도 고표본을 발굴하여 기존의 고표본 $\mathrm{DB}$ 를 확장 및 보완하는 데에 목적이 있다.

\section{재료 및 방법}

2018년 8월 5일에서 8월 25일까지, 2019년 2월 11일에 서 2월 28일까지 도쿄대학 박물관에 체류하면서 동정 및 라벨 작업을 하고, 중복채집품(duplicate)을 확보하였다. 익숙하지 못한 분류군은 도쿄대 표본관에 소장되어 있는

*Author for correspondence: ihtplant@hanmail.net Open Access http://e-kjpt.org, (C) 2019 the Korean Society of Plant Taxonomists. This is an open-access article distributed under the terms of the Creative Commons Attribution Non-Commercial License
(http://creativecommons.org/licenses/by-nc/4.0) which permits unrestricted non-commercial use, distribution, and reproduction in any medium, provided the original work is properly cited. 
기동정 표본을 참조하여 동정을 진행했다. 생식기관이 없는 불완전한 표본의 경우 속수준의 동정을 했다.

\section{결과 및 고찰}

\section{최근 확인된 T의 미동정 한반도 고표본}

도쿄대학 표본관의 미동정 한반도 고표본은 이름이 알 려진 식물분류학자 및 채집자의 표본이 대부분으로, $\mathrm{T}$. Nakai와 T. Uchiyama의 표본이 주를 이루었으며 일부 T. Mori와 T. Ishidoya의 표본이 들어있었다. 미동정 고표본 중에는 비전공자인 일반인들에 의해 채집된 표본들도 있 었으며, 이 표본들은 문헌 정보에도 인용되지 않은 경우 가 대부분이었다. 이중에는 구한말 일본 공사를 지냈던 Hanabusa Yoshimoto (花房 義質)와 같은 유명인(Fig. 1C) 은 물론 Tsuyama Toshizo (津谷 俊三)와 같은 무명의 공무 원(Fig. 1D) 등이 있었으며, 곤충학자인 석주명선생이 함 흥에서 채집한 약 100 종의 식물표본도 도쿄대학에 소장 되어 있었다. Saito Siroji (齊藤 四郎治)의 채집품처럼 체 계적으로 여러 지역에서 많은 식물을 채집한 경우에 대 해서는 선행연구에서 소개한 바 있다(Im et al., 2016).

고표본 라벨의 채집지 기록은 당시의 한반도 현지 지 명 발음을 일본글(카나) 또는 알파벳으로 표기한 것이 일 반적이었다. 한자로 표기된 지명 중에도 현재의 지명과 다른 경우가 있어서 채집지를 특정하기 힘들었으며, 북 한 지역은 새롭게 개명된 곳이 의외로 많아서 일본 연구 자들은 물론 우리도 고표본의 라벨 정보를 이용하기 쉽 지 않은 경우가 있었다. 일반적으로 고표본에는 손으로 쓴 라벨이라도 들어 있지만, 때로는 라벨이 없이 채집지 또는 채집일만 신문지에 수기되어 있는 경우도 있었다 (Fig. 1A, B). Fig. 1A는 Saito Siroji (齊藤 四郎治)의 채집품 으로 표본 뭉치의 표지 신문에 "1926년 6월 19일 조선 Kannonsan (観音山)"으로 수기되어 있었고, 첨부된 라벨 에 Bentenzan (弁天山)으로 표기되어 있었다. 일본에서는 관음보살을 변천(弁天)으로 부르기도 하므로 이들 표본 이 채집된 Kannonsan (観音山) 또는 Bentenzan (弁天山)은 거제도에 있는 관음사 뒷산인 옥녀봉을 지칭한 것으로 추정되었다. Fig. $1 \mathrm{~B}$ 는 신문지 여백에 “加 8,12 ”라는 수기 이외에 어떤 정보도 제시되어 있지 않았다. 기존 고표본 $\mathrm{DB}$ 에서 “伽 8,12 ”를 주요어(key word)로 검색한 결과 이 들 표본은 T. Nakai가 1919년 8월 12일 가야산에서 채집한 표본임을 확인할 수 있었다. 이와 같이 한반도 고표본 확 보 작업은 기존 라벨(일문 라벨)이 있는 경우에도 우리나 라 현재 지명을 특정하여 영문 라벨을 새로 만든 다음, 동 정을 하는 수순으로 진행하였다. 이번 조사에서 확보된 한반도 고표본 중에는 기존의 고표본 $\mathrm{DB}$ 에 올라있지 않 는 표본이 상당수 있었으며, 그 중 Ikuma Yoichiro (生熊 与一郎)의 채집품은 한반도 북동지역에서 체계적으로 채집되었다.

\section{Ikuma Yoichiro (生熊 与一郎)의 채집품}

Y. Ikuma는 곤충학자로 『응용곤충학교과서, 1904년』, 『동물표본 및 모형제작법, 1913년』과 같은 곤충학 저서 를 남겼으며, 석주명 선생처럼 곤충조사를 하면서 식물 채집도 했던 것으로 추정된다. Y. Ikuma의 채집품중 일부 는 기존 고표본 $\mathrm{DB}$ 에 이미 등재되어 있으나, 표본 정보에 두 군데 오류가 있는 것으로 보였다. 먼저 채집자 이름이 Ikumatsu I.로 되어 있는데, 이는 고표본 라벨 정보를 DB 화하면서 채집자의 성(生熊)을 읽고 표기하는 과정에서 생긴 착오로 생각된다. 다음으로 채집년월이 일부는 1913년 8월로 나머지는 1914년 8월로 되어 있는데, 표본 에 적힌 원정보를 라벨화하는 과정에서 오류가 발생한 것으로 추정된다(Fig. $1 \mathrm{E}, \mathrm{F})$. Fig. 1E는 무두봉, 백두산, 신 민둔에서 채집된 표본 뭉치의 표지로서 신문지 왼쪽 여 백에 신민둔 8, 14로 표기되어 있고, 다른 필체로 1914(3) 로 표기되어 있어서 1914년(혹은 1913년) 8월 14일 채집 되었음을 알 수 있다. 기존 고표본 DB의 Y. Ikuma 채집품 은 1913년과 1914년 모두 7월 31일부터 8월 23일까지의 기간 동안에 무산, 농사동, 백두산, 혜산진 지역에서 채집 된 것으로 두 해에 걸쳐서 채집되었다고 보기에는 무리 가 있다. Y. Ikuma가 TI로 보낸 채집품의 일부를 도쿄대 연구자들이 표본화하는 과정에서 기재 오류가 생긴 것으 로 추정된다. Fig. $1 \mathrm{~F}$ 는 미동정 상태로 대지에 올려진 고 표본의 라벨인데, 아래쪽 표본은 “咸南 惠山鎭 Aug. 23. 1914. 中井猛之進”, 위쪽 표본은 “咸南 茂山農事洞 Aug. 8. 1913. 生熊与一朗"으로 표기되어 있다. 이 라벨의 필적 은 양쪽 모두 T. Nakai 박사의 글씨체로 추정되는데, T. Nakai 박사의 기존 조사 행적에 의하면 Aug. 23. 1914.에 는 함북 회령에서 채집했던 것으로 확인된다. 더욱이 위 쪽 표본에는 채집자명을 中井이라고 썼다가 中을 生으로 고치고, 井 대신에 熊을 기입한 것으로 보인다. 그러므로 아래쪽 표본의 라벨 정보는 잘못된 것으로, 이 표본은 $\mathrm{T}$. Nakai라 아니라 Y. Ikuma의 채집품일 가능성이 높다. 신 문지의 채집 연도(1914 혹은 1913)와 TI 라벨의 기재 내용, 그리고 기존 고표본 DB의 Y. Ikuma 표본 중에 1914년보 다 1913년 채집품이 많은 점을 종합적으로 고려하여, 본 연구에서는 Y. Ikuma 채집품의 채집년도를 1913년으로 추정했다.

이번 연구에서 확보된 Y. Ikuma의 채집품은 당시의 행 정구역으로 함경북도와 함경남도 지역에서 채집되었는 데, 해방 후 백두산과 혜산진 지역이 양강도로 개편되었 기 때문에 현재로서는 함경북도와 양강도 지역에 해당한 다(Fig. 2A). 이 표본들은 1913년 8월에 청진(淸津)-차유 령(車踰嶺)-무산(茂山)-강구(江口)-농사동(農事洞)-무 봉(茂峰)-무두봉(茂頭峰)- 백두산(白頭山) - 최가령(崔可 嶺)-혜산진(惠山䤾) 지역에서 채집되었다(Fig. 2, Table 1). 이 지역들은 한반도의 대표적인 고산지대(백두산-개 마고원지역)의 일부로서 한대-아한대식물의 분포중심지 

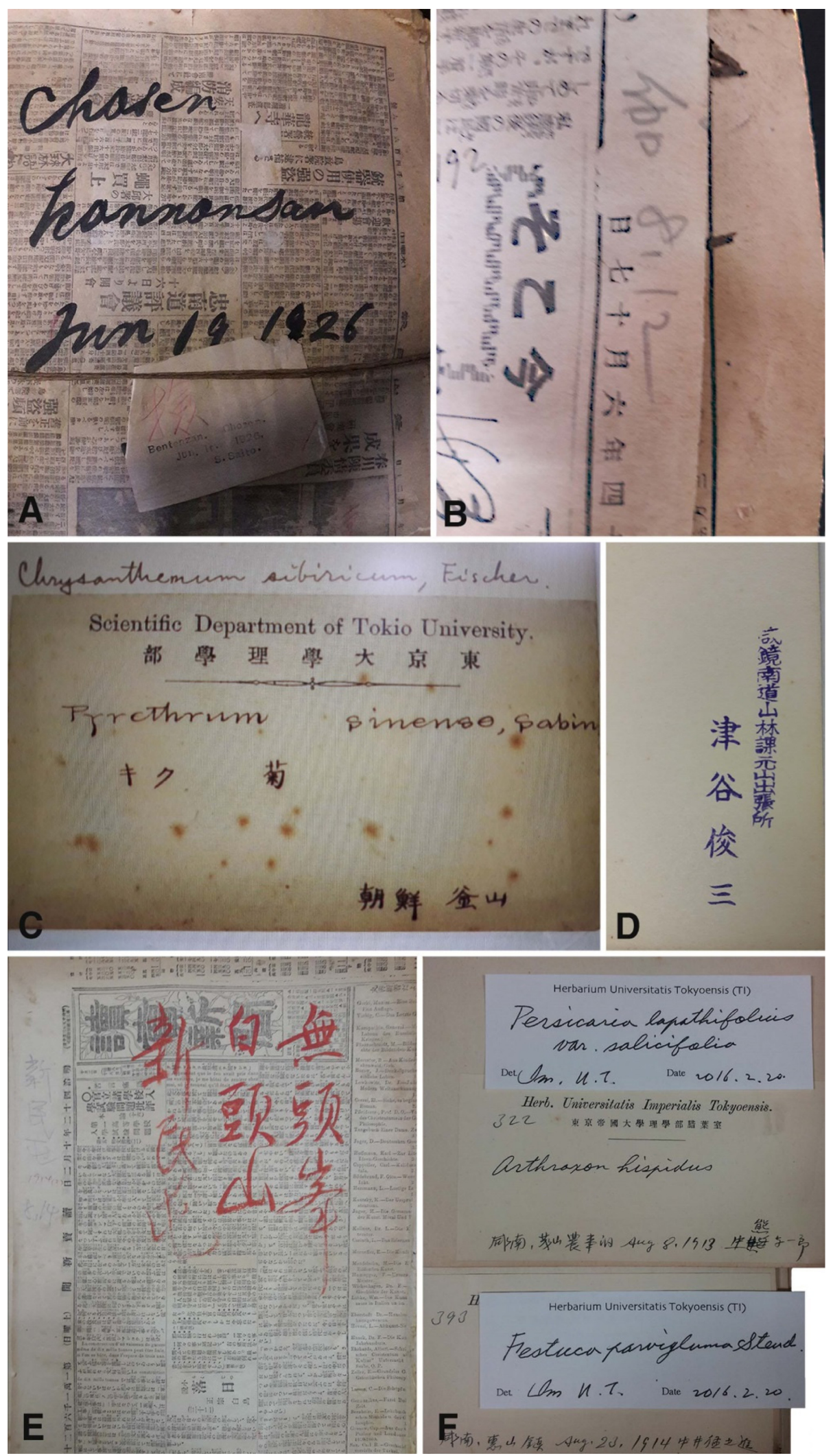

Fig. 1. A. Handwriting collecting data by S. Siroji. B. Unclear collecting data by T. Nakai. C. Label of Y. Hanabusa, the first minister of Japan. D. Label of T. Tsuyama, a provincial government official. E. Handwriting collecting data by Y. Ikuma. F. Unclear collecting data had resulted from mistaken labeling of some Y. Ikuma collection. 

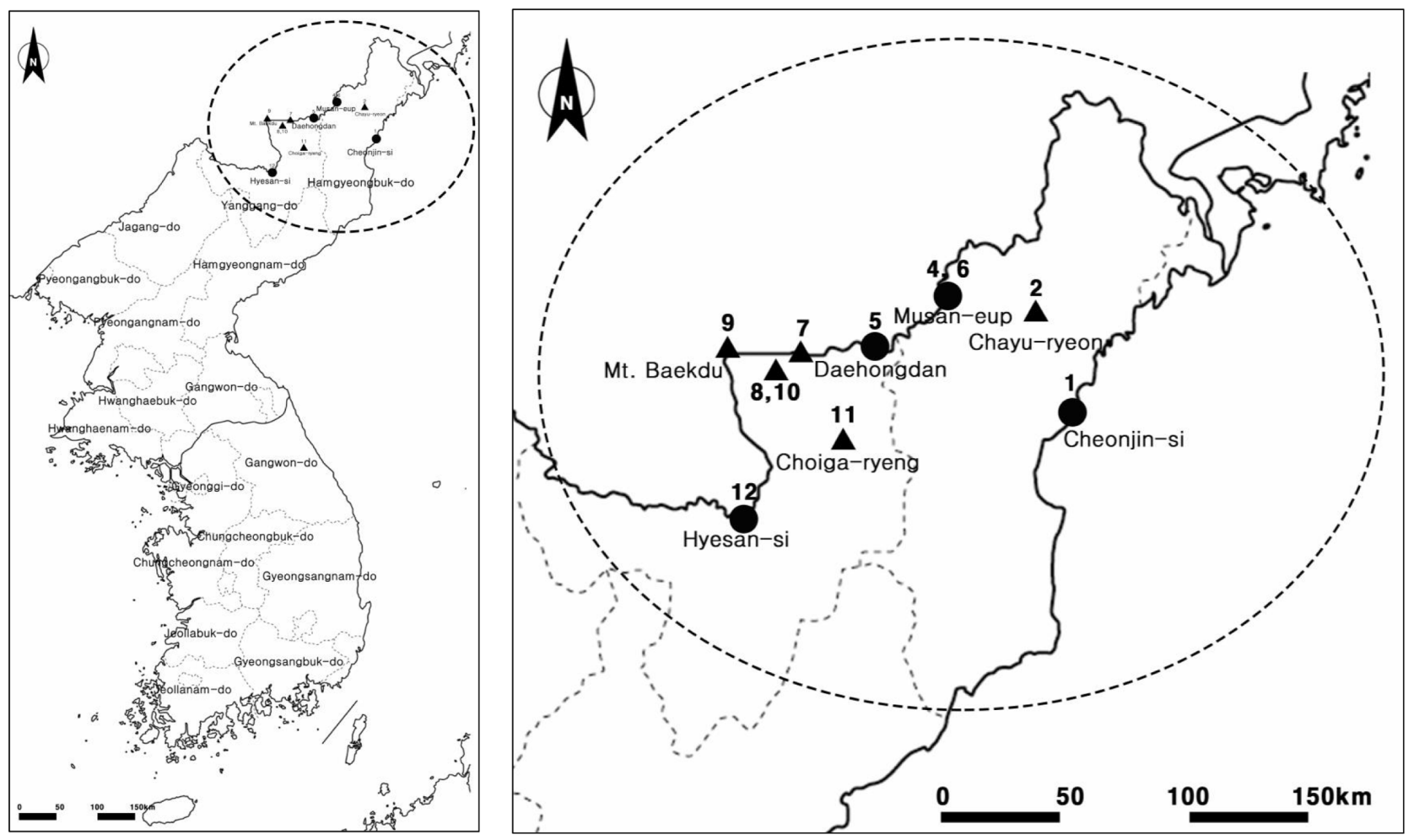

Fig. 2. Major sites of the Y. Ikuma collections in the Korean Peninsula. 1, Cheongjin-si; 2, Chayu-ryeong; 3, Musan; 4, Musan Ganggu; 5, Daehongdan; 6, Musan Ganggu; 7, Mubong; 8, Mudu-bong; 9, Baekdu-san; 10, Mudu-bong; 11, Choiga-ryeong; 12, Hyesan-si.

Table 1. Collection dates and locations of newly found historic specimens among Y. Ikuma (生熊 与一郎) collections.

\begin{tabular}{ccc}
\hline \hline No. & Date & Location \\
\hline 1 & 1 Aug. 1913 & Hamgyeongbuk-do, Cheongjin \\
2 & 2-3 Aug. 1913 & Hamgyeongbuk-do, Bureyong-gun, Chayu-ryeong (pass, $914 \mathrm{~m}$ elev.) \\
3 & 5 Aug. 1913 & Hamgyeongbuk-do, Musan-gun \\
4 & 7 Aug. 1913 & Hamgyeongbuk-do, Musan-gun, Ha-myeon, Ganggu \\
5 & 8 Aug. 1913 & Ryanggang-do, Daehongdan-gun, Nongsa-dong \\
6 & 9 Aug. 1913 & Hamgyeongbuk-do, Musan-gun, Ha-myeon, Ganggu \\
7 & 10 Aug. 1913 & Hamgyeongbuk-do, Musan-gun, Mu-bong (peak, 1,320 m elev.) \\
8 & 12 Aug. 1913 & Ryanggang-do, Samjiyeon-gun, Sinmuseong/Mudu-bong (peak, 1,930 m elev.) \\
9 & 13 Aug. 1913 & Ryanggang-do, Samjiyeon-gun, Baekdu-san Mt. (2,750 m alt) \\
10 & 14 Aug. 1913 & Ryanggang-do, Samjiyeon-gun, Sinmuseong/Mudu-bong (peak, 1,930 m elev.) \\
11 & 19-21 Aug. 1913 & Ryanggang-do, Bocheon-gun, Choiga-ryeong (pass, 1,605 m elev.) \\
12 & 22-23 Aug. 1913 & Ryanggang-do, Hyesan-gun, Hyesanjin \\
\hline
\end{tabular}

이다. 주로 백두산에서 촬영된 희귀 북방계식물의 사진 은 국내 도감에 많이 실려 있지만, 국내에 북한산 표본이 거의 없는 현재 상황에서 이 연구에서 확보된 중복채집 품(duplicate) 및 라벨 정보는 한반도의 식물상 및 식물분 포 연구에 있어서 중요한 기초자료로 활용될 수 있을 것
으로 생각한다. 표본으로 확보된 Y. Ikuma의 채집품은 60 과 177속 240종(미동정 3분류군 포함)으로 정리되었다 (Appendix 1). 부록에 제시된 목록은 과명, 학명, 국명, 채 집지의 순으로 배열했으며, 채집지 번호는 Table 1의 채 집 날짜와 동일하다. 


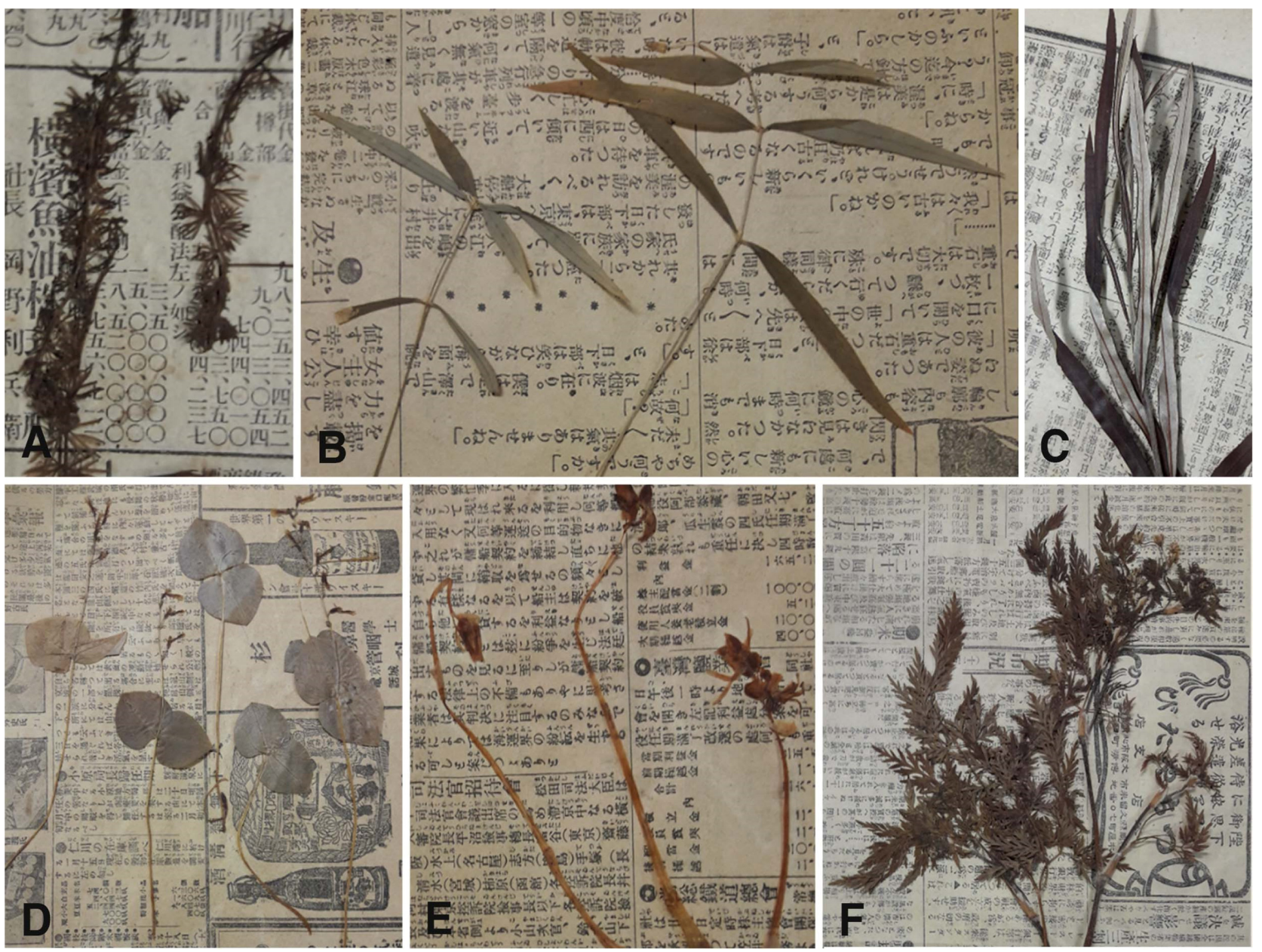

Fig. 3. Important specimens from the Y. Ikuma collections in NE part of Korean Peninsula. A. Urticularia intermedia Hayne. B. Stellaria longifolia Muhl. ex Willd. C. Salix viminalis L. D. Listera pinetorum Lindl. E. Epipogium aphyllu Sw. F. Libanotis coreana (H. Wolff) Kitagawa

\section{중요식물}

이번에 추가로 확보된 Y. Ikuma의 채집품 중 특히 의미 있는 식물들은 백두산과 개마고원을 중심으로 한 고산지 대에 제한 분포하는 식물들이다.

1. 개통발(Urticularia intermedia Hayne (DC.) J. R. Grant) (Fig. 3A)

개통발은 땅속으로 벋는 줄기에 포충낭이 달리며, 호 생하는 잎은 주축이 없이 몇 번 차상분지 하는 점이 통발 또는 들통발과 구분되는 특징이다. Y. Ikuma의 표본은 최 가령에서 채집되었는데, 생식기관이 없는 불완전한 상태 이지만 여러 번 차상분지하는 잎이 호생하고 있어서 개 통발로 동정했다. 북반구의 아한대와 냉온대를 중심으로 넓게 분포하는 수생식충식물로 우리나라에는 백두산을 중심으로 한 고산지대에 자라며, 강원도 대암산에서도 확인되었다.
2. 긴잎별꽃(Stellaria longifolia Muhl. ex Willd., Fig. 3B) 별꽃속 중 가늘고 긴 선형의 잎을 가진 분류군은 실별 꽃과 긴잎별꽃이 있으며, 긴잎별꽃은 줄기 상부 능선에 미세한 돌기가 발달하는 특징이 있다. 최가령에서 채집 된 이 표본은 잎폭이 3-4 mm로서 기재문에 비해 넓지만 줄기 상부 능선에 미세한 돌기가 있어서 긴잎별꽃으로 동정했다. 북반구 고위도지방에 널리 분포하는 전형적인 주북극요소 식물로 한반도에는 백두산과 개마고원지역 에 자라며, 경기도 수원 부근에도 분포하는 것으로 알려 져 있다.

\section{3. 육지꽃버들(Salix viminalis L.) (Fig. 3C)}

육지꽃버들은 잎이 선상 피침형으로 $10-15 \mathrm{~cm} \times 0.3-$ $1 \mathrm{~cm}$ 이고, 아랫면에 은색 융모가 밀생하며, 자방이 밀모로 덮여 있고, 암술머리는 옅게 둘로 나뉘는 특징이 있다. 최 가령에서 채집된 이 표본은 생식기관이 없는 불완전한 상 
태이지만, 아랫면에 은색 융모가 밀생한 긴 선상피침형의 잎을 기준으로 동정했다. 극동러시아, 중국 동북지방에 분 포하며, 한반도에는 압록강과 두만강 주변 지역에 자라고 국내에는 해방 전에 채집된 표본이 몇 점 있다.

4. 쌍잎난초(Neottia pinetorum (Lindl.) Szlach.) (Fig. 3D) 한 쌍의 잎이 대생하는 독특한 외관의 지생란으로 히 말라야, 중국 동북지방, 극동러시아에 분포한다. 한반도 에는 백두산과 개마고원지역의 냉온대 침엽수림에 주로 자란다. Y. Ikuma의 채집품 중에는 강구와 최가령에서 각 각 채집되었다.

\section{5. 유령란(Epipogium aphyllum Sw., Fig. 3E)}

잎도 없고, 엽록소도 없는 부생란으로 유라시아의 냉온 대 및 아고산대 침엽수림에 주로 분포하며, 한반도에는 백두산을 중심으로 자란다. 일본의 유령란은 절멸위기종 $1 \mathrm{~B}$ 류$(\mathrm{EN})$ 로서 Hokkaido에서 Chubu 지방의 아고산대로 점 점이 분포하지만, 근연종인 E. roseum (D. Don) Lindl.은 반 대로 열대지방에 기원하여 Kyushu를 거쳐 Kanto 지방까 지 북상하는 분포양상을 보인다(Yamamoto et al., 2017).

6. 털기름나물(Libanotis coreana (H. Wolff) Kitag (Fig. 3F) 털기름나물은 유라시아대륙에 분포하며, 한반도에는 백두산과 한라산에 격리분포하는 것으로 알려져 있으나, 가는잎방풍(Libanotis seseloides (Fisch. \& C. A. Meyer. ex Turcz.) Turcz.)에 포함시키는 견해도 있다. 혜산진에서 채 집된 이 표본은 전체에 털이 있고, 잘 발달된 엽초와 총포 가 특징적이다.

\section{7. 주북극요소(circumpolar element) 식물}

유라시아와 북아메리카의 한대 및 아한대지역에 넓게 분포하는 대표적인 북방계식물로 한반도의 경우, 백두산 과 개마고원 지역이 주분포지이다. 중부 이남에는 설악 산, 한라산 등 고산지대에 격리분포하는데, 빙기에 남쪽 으로 세력을 확장했다가 간빙기의 온난화에 의해 고산지 대에 유존하게 된 것으로 추정된다. Y. Ikuma의 채집품 중 에는 홍월귤(Arctous ruber (Rehder \& E. H. Wilson) Nakai), 개병풍(Astilboides tabularis (Hemsl.) Engl.), 독미나리 (Cicuta virosa L.), 닻꽃(Halenia corniculata (L.) Cornaz), 이 삭단엽란(Microstylis monophyllos (L.) Sw.), 애기사철란 (Goodyera repens (L.) R. Br.), 나도범의귀(Mitella nuda L.) 등이 이에 해당한다.

ORCID: Eun-Mi SUN https://orcid.org/0000-0001-76918094; Kae Sun CHANG https://orcid.org/0000-0003-44546059; Hyun-Duk SON https://orcid.org/0000-0002-35041908; Hyoung-Tak IM https://orcid.org/0000-0002-63336244.

\section{Acknowledgments}

This research was funded by Korea National Arboretum KNA1-1-23, 18-1 (the development of online flora-based research to enhance understanding of plant species diversity in the Korean Peninsula). We sincerely thank Dr. Jin Murata and Dr. Hirosi Ikeda for allowing full use of the herbarium facilities of the University of Tokyo. Botanical works of Dr. Chang Chin-Sung (the botanical gazetteer for Korean Peninsula and a catalogue of vascular plant type specimens from Korea) contributed greatly to the preparation and presentation of this paper.

\section{Conflict of Interest}

The authors declare that there are no conflicts of interest.

\section{Literature Cited}

Chang, C.-S. and K. S. Chang. 2010. Reexamination on foreign collectors' sites and exploration routes in Korea (IV): with respect to T. Ishidoya. Korean Journal of Plant Taxonomy 40: 90-104. (in Korean)

Chang, C.-S., B.-H. Choi, H. Kim and J.-Y. Lee. 2004. Reexamination on foreign collectors' sites and exploration routes in Korea: with respect to U. Faurie. Korean Journal of Plant Taxonomy 34: 87-96. (in Korean)

Chang, K.-S. S.-K. Park, H. Kim and C.-S. Chang. 2013. A gazetteer of three Japanese taxonomists (G. Koidzumi, J. Ohwi, and S. Kitamura) of Kyoto University in Korea during 1930s. Korean Journal of Plant Taxonomy 43: 319-331. (in Korean)

Im, H.-T., H.-D. Son and J.-S. Im. 2016. Historic plant specimens collected from the Korean Peninsula in the early 20th century (I). Korean Journal of Plant Taxonomy 46: 33-54.

Kim, H., G.-S. Chang, C.-S. Chang and B.-H. Choi. 2006. Reexamination on foreign collectors' sites and exploration routes in Korea (II): with respect to T. Nakai. Korean Journal of Plant Taxonomy 36: 227-255. (in Korean)

Kim, H., K. S. Chang, H. Y. Gil, S. K. Park and C.-S. Chang. 2012. T. Mori's collections and resolving place names in Korea. Korean Journal of Plant Taxonomy 42: 340-353. (in Korean)

Kim, H., B.-H. Choi, C.-S. Chang and K.-S. Chang. 2007. Reexamination on foreign collectors' sites and exploration routes in Korea (III): with respect to T. Uchiyama. Korean Journal of Plant Taxonomy 37: 203-215.

Yamamoto, N., M. Amano, H. Ikeda and H. T. Im. 2017. A note for Epipogium roseum (Orchidaceae) collected in Minamiboso city. Flora of Chiba 65: 43-39. (in Japanese) 


\section{0세기초에 채집된 한반도 고표본 (II) \\ 선은미 · 장계선 · 손현덕 ${ }^{1}$ 임형탁 ${ }^{2 *}$}

국립수목원 산림생물다양성연구과, ${ }^{1}$ 목포자연사박물관, ${ }^{2}$ 전남대학교 자연과학대학 생물학과

적 요: 20 세기초 한반도에서 채집된 고표본들은 한국전쟁 동안 대부분이 소실되었으나, 일본 도쿄대학교 표본 관(TI)에 소장된 한반도 고표본들은 온전하게 보존되어 있다. 북한 식물에 대한 정보를 얻을 수 없는 현 상황에 서 $\mathrm{TI}$ 의 한국산 고표본 정보는 매우 중요하다. TI 수장고에서 최근 확인된 미동정 상태의 한반도 고표본을 동정 하여 목록을 작성했으며, 중복채집품(duplicate)을 확보했다. 곤충학자인 Ikuma Yoichiro는 1913년 8월 1일부터 23 일까지 청진에서 백두산을 거쳐 혜산진에 이르는 일정 동안 60 과 177 속 240 종류의 식물을 채집했다.

주요어: 한반도 고표본, 20 세기 초, 도쿄대학 표본관, 이쿠마 요이치로, 희귀식물

Appendix 1. Historic specimens collected by Y. Ikuma in Aug 1913 in the northeastern part of Korean Peninsula.

\begin{tabular}{|c|c|c|c|c|c|c|c|c|c|c|c|c|c|c|}
\hline \multirow{2}{*}{ Family } & \multirow{2}{*}{ Taxa } & \multicolumn{13}{|c|}{ Collection date } \\
\hline & & 1 & 2 & 7 & 8 & 9 & 10 & 12 & 13 & 14 & 19 & 20 & 2122 & 23 \\
\hline Selaginellaceae & Selaginella rossii (Baker) Warb. 구실사리 & $\bigcirc$ & & & & & & & & & & & & \\
\hline \multirow[t]{2}{*}{ Equisetaceae } & Equisetum ramosissimum Desf. 개속새 & & & & & & & & & & & $\bigcirc$ & & \\
\hline & Equisetum sylvaticum L. 능수쇠뜨기 & & & & & & ○ & & & & & & & \\
\hline Pteridaceae & Cheilanthes argentea (S. G. Gmel.) Kunze 부시깃고사리 & $\bigcirc$ & & & & & & & & & & & & \\
\hline Aspleniaceae & Athyrium yokoscense (Franch. \& Sav.) Christ 뱀고사리 & & & & & $\bigcirc$ & & & & & & & & \\
\hline Woodsiaceae & Woodsia ilvensis (L.) R. Br. 두메우드풀 & & & $\bigcirc$ & & & & & & & & & & \\
\hline Athyriaceae & Athyrium vidalii (Franch. \& Sav.) Nakai 산개고사리 & & & & & & & & & & & & & $\bigcirc$ \\
\hline Davalliaceae & Thelypteris torresiana var. calvata (Baker) K. Iwats. 각시고사리 & $\bigcirc$ & & & & & & & & & & & & \\
\hline \multirow[t]{2}{*}{ Polypodiaceae } & Lepisorus ussuriensis (Regel \& Maack) Ching 산일엽초 & $\bigcirc$ & & & & & & & & & & & & \\
\hline & Pyrrosia petiolosa (Christ) Ching 애기석위 & & & $\bigcirc$ & & & & & & & & & & \\
\hline Cupressaceae & Juniperus rigida Siebold \& Zucc. 노간주나무 & & & & & & & & & $\bigcirc$ & & & & \\
\hline \multirow[t]{13}{*}{ Ranunculaceae } & Aconitum jaluense Kom. 투구꽃 & & & & & & & & & & & & & $\bigcirc$ \\
\hline & Aconitum longecassidatum Nakai 흰진범 & & & & & & & & & & & & & $\bigcirc$ \\
\hline & Aconitum sibiricum Poir. 노랑투구꽃 & $\bigcirc$ & & & & & & & & & & & & \\
\hline & Cimicifuga heracleifolia Kom. 승마 & $\bigcirc$ & & & & & & & & & & & & \\
\hline & Cimicifuga simplex (DC.) Turcz. 촛대승마 & & & & & & & & & & & & & $\bigcirc$ \\
\hline & Clematis hexapetala Pall. 좁은 잎사위질빵 & O & & & & & & & & & & & & \\
\hline & Clematis trichotoma Nakai 할미밀 망 & & $\bigcirc$ & & & & & & & & & & & \\
\hline & Enemion raddeanum Regel 나도바람꽃 & & & & & & & & & & & & $\bigcirc$ & \\
\hline & Ranunculus franchetii $\mathrm{H}$. Boissieu 왜미나리아재비 & & & & & & & & & $\bigcirc$ & & & & \\
\hline & Ranunculus japonicus Thunb. 미나리아재비 & & & & & & & & & & $\bigcirc$ & & & \\
\hline & Thalictrum actaeifolium var. brevistylum Nakai 은뀡 의다리 & $\bigcirc$ & & & & & & & & & & & & \\
\hline & Thalictrum sp. & & & & & & & & & $\bigcirc$ & & & & \\
\hline & Thalictrum tuberiferum Maxim. 산꿩의다리 & & & & & & & & & & & & & $\bigcirc$ \\
\hline Berberidaceae & Epimedium koreanum Nakai 삼지구엽초 & & & & & & & & & & & & & $\bigcirc$ \\
\hline \multirow[t]{2}{*}{ Papaveraceae } & Chelidonium majus var. asiaticum (Hara) Ohwi 애기똥풀 & & & & & & & & & & & & & $\bigcirc$ \\
\hline & Papaver coreanum Nakai 두메양귀비 & & & & & & & & $\bigcirc$ & & & & & \\
\hline
\end{tabular}


Table 1. Continued.

\begin{tabular}{|c|c|c|c|c|c|c|c|c|c|c|c|c|c|c|}
\hline \multirow{2}{*}{ Family } & \multirow{2}{*}{ Taxa } & \multicolumn{13}{|c|}{ Collection date } \\
\hline & & 1 & 2 & 7 & 8 & 9 & 10 & 12 & 13 & 14 & 19 & 20 & 2122 & 23 \\
\hline Ulmaceae & Ulmus davidiana Planch. 당느릅나무 & & O & & & & & & & & & & & \\
\hline Urticaceae & Urtica angustifolia Fisch. ex Hornem. 가는잎쐐기풀 & $\bigcirc$ & & & & & & & & & & & & \\
\hline Fagaceae & Quercus mongolica Fisch. ex Ledeb. 신갈나무 & $\bigcirc$ & & & & & & & & & & & & $\bigcirc$ \\
\hline Aristolochiaceae & Asarum sieboldii Miq. 족도리풀 & & & & & & & & & & & $\bigcirc$ & & \\
\hline Chenopodiaceae & Chenopodium album var. centrorubrum Makino 명아주 & & & & & & & & & & & & & $\bigcirc$ \\
\hline \multirow[t]{6}{*}{ Caryophyllaceae } & Dianthus chinensis var. chinensis L. 패랭이꽃 & $\bigcirc$ & & & & & & & & & & & & \\
\hline & Gypsophila pacifica Kom. 가는대나물 & $\bigcirc$ & & & & & & & & & & & & \\
\hline & Silene foliosa Maxim. 호산장구채 & $\bigcirc$ & & & & & & & & & & & & \\
\hline & Stellaria aquatica (L.) Scop. 쇠별꽃 & & & & & & & & & & & & & $\bigcirc$ \\
\hline & Stellaria longifolia Muhl. ex Willd. 긴잎별꽃 & & & & & & & & & & $\bigcirc$ & & & \\
\hline & Stellaria radians L. 왕별꽃 & & & & & & & & & & & & & O \\
\hline \multirow[t]{6}{*}{ Polygonaceae } & Aconogonon microcarpum (Kitag.) H. Hara 참개싱아 & $\bigcirc$ & & & & & & & & & & & & \\
\hline & Fallopia dentatoalata (F. Schmidt) Holub 큰닭의덩굴 & & & & & & & & & & & & & O \\
\hline & Persicaria nepalensis (Meisn.) H. Gross 산여뀌 & & & & & & & & & & & & & $\bigcirc$ \\
\hline & Polygoinatum lapathifolium L. 명아자여뀌 & & & & & & & & $\bigcirc$ & & & & & \\
\hline & Polygonum aviculare L. 마디풀 & & & & & & & & & & & & & $\bigcirc$ \\
\hline & Polygonum hydropiper (L.) Delarbre 여뀌 & & & & & & & & & & & & $\bigcirc$ & \\
\hline Clusiaceae & Hypericum ascyron L. 물레나물 & & & & & & & & & & & & & $\bigcirc$ \\
\hline Tiliaceae & Tilia amurensis Rupr. 피나무 & $\bigcirc$ & & & & & & & & & & & & \\
\hline \multirow[t]{2}{*}{ Salicaceae } & Salix rorida Laksch. 분버들 & & O & & & & & & & & & & & \\
\hline & Salix viminalis L. 육지꽃버들 & & & & & & & & & & & & $\bigcirc$ & \\
\hline \multirow[t]{2}{*}{ Brassicaceae } & Arabis pendula L. 느러진장대 & & & & & & & & & & & & & $\bigcirc$ \\
\hline & Cardamine leucantha (Tausch) O.E. Schulz 미나리냉이 & & & & & & & & & & & & & $\bigcirc$ \\
\hline Pyrolaceae & Pyrola dahurica (H. Andres) Kom. 호노루발 & & & & & & & & & & & & & $\bigcirc$ \\
\hline \multirow[t]{4}{*}{ Ericaceae } & Arctous ruber (Rehder \& E.H. Wilson) Nakai 홍월귤 & & & & & & $\bigcirc$ & & & & & & & \\
\hline & Rhododendron dauricum L. 산진달래나무 & & & & & & $\bigcirc$ & & & & & & & \\
\hline & Rhododendron parviflorum F. Schmidt 황산차 & & & & & & $\bigcirc$ & & & & & & & \\
\hline & Rhododendron redowskianum Maxim. 좀참꽃 & & & & & & & & $\bigcirc$ & & & & & \\
\hline \multirow[t]{3}{*}{ Primulaceae } & Androsace septemtrionalis L. 명천봄맞이 & & & & & & & & & $\bigcirc$ & & & & \\
\hline & Androsace umbrella (Lour.) Merr. 봄맞이 & & & & & & & & & & & & $\bigcirc$ & \\
\hline & Lysimachia vulgaris var. davurica (Ledeb.) R. Kunth 좁쌀풀 & $\bigcirc$ & & & & & & & & & & & & \\
\hline Crassulaceae & Hylotelephium telephium (L.) H. Ohba 자주꿩의비름 & & $\bigcirc$ & & & & & & & & & & & \\
\hline \multirow[t]{6}{*}{ Saxifragaceae } & Astilbe rubra Hook.f. \& Thomson 노루오줌 & & O & & & & & & & & & & & \\
\hline & Astilboides tabularis (Hemsl.) Engl. 개병풍 & & & & & & & & & & & & & $\bigcirc$ \\
\hline & Mitella nuda L. 나도범의귀 & & & & & & & & & & & & $\bigcirc$ & \\
\hline & Parnassia palustris L. 물매화 & & & & & & & & & & & & & $\bigcirc$ \\
\hline & Ribes mandshuricum (Maxim.) Kom. 까치밥나무 & & & & & & & & & & & & $\bigcirc$ & \\
\hline & Saxifraga manchuriensis (Engl.) Kom. 흰바위취 & & $\bigcirc$ & & & & & & & & & & & \\
\hline \multirow[t]{2}{*}{ Rosaceae } & Agrimonia pilosa Ledeb. 짚신나물 & $\bigcirc$ & & & & & & & & & & & & $\bigcirc$ \\
\hline & Crataegus pinnatifida Bunge 산사나무 & $\bigcirc$ & & & & & & & & & & & & \\
\hline
\end{tabular}


Table 1. Continued.

\begin{tabular}{|c|c|c|c|c|c|c|c|c|c|c|c|c|c|}
\hline \multirow{2}{*}{ Family } & \multirow{2}{*}{ Taxa } & \multicolumn{12}{|c|}{ Collection date } \\
\hline & & 1 & 2 & 7 & 8 & 9 & 10 & 12 & 13 & 1419 & 20 & 2122 & 23 \\
\hline \multirow[t]{13}{*}{ Rosaceae } & Duchesnea sp. & & & & & & & & & $\bigcirc$ & & & \\
\hline & Geum aleppicum Jacq. 큰뱀무 & $\bigcirc$ & & & & & & & & & & & \\
\hline & Potentilla centigrana Maxim. 좀딸기 & & & & & & & & & & & & $\bigcirc$ \\
\hline & Potentilla chinensis Ser. 딱지꽃 & $\bigcirc$ & & & & & & & & & & & \\
\hline & Potentilla cryptotaeniae Maxim. 물양지꽃 & & & & & & & & & & & & $\bigcirc$ \\
\hline & Potentilla fruticosa var. rigida (Wall.) Th. Wolf 물싸리 & & & & $\bigcirc$ & & & & & & & & \\
\hline & Prunus maackii Rupr. 개벚지나무 & & & & & & & & & & & & $\bigcirc$ \\
\hline & Rosa acicularis Lindl. 인가목 & & & & & & & & $\bigcirc$ & & & & \\
\hline & Rosa davurica Pall. 생 열귀나무 & $\bigcirc$ & & & & & & & & & & & \\
\hline & Sanguisorba longifolia Bertol. 긴오이풀 & & & & & & & & $\bigcirc$ & & & & \\
\hline & Sorbaria sorbifolia var. stellipila Maxim. 쉬땅나무 & & & & & & & & & & & & $\bigcirc$ \\
\hline & Spiraea pubescens Turcz. 아구장나무 & & $\bigcirc$ & & & & & & & & & & \\
\hline & Spiraea salicifolia L. 꼬리조팝나무 & & $\bigcirc$ & & & & & & & & & & O \\
\hline \multirow[t]{12}{*}{ Fabaceae } & Astragalus membranaceus Bunge 황기 & & & & & & & & & & & & $\bigcirc$ \\
\hline & Hedysarum alpinum L. 묏황기 & & $\bigcirc$ & & & & & & & & & & \\
\hline & Lathyrus davidii Hance 활량나물 & & & & & & & & & & & & $\bigcirc$ \\
\hline & Lespedeza tomentosa (Thunb.) Siebold ex Maxim. 개싸리 & & $\bigcirc$ & & & & & & & & & & \\
\hline & Melilotus suaveolens Ledeb. 전동싸리 & & $\bigcirc$ & & & & & & & & & & \\
\hline & Sophora flavescens Solander ex Aiton 고삼 & $\bigcirc$ & & & & & & & & & & & \\
\hline & Thermopsis lupinoides (L.) Link 갯활량나물 & & & & & & & & & & & & $\bigcirc$ \\
\hline & Trifolium lupinaster L. 달구지풀 & & & & & & & & & & & & $\bigcirc$ \\
\hline & Vicia amurensis Oett. 벌완두 & $\bigcirc$ & $\bigcirc$ & & & & & & & & & & \\
\hline & Vicia cracca L. 등갈퀴나물 & & & & & & & & & & & & $\bigcirc$ \\
\hline & Vicia unijuga A. Braun 나비나물 & & $\bigcirc$ & & & & & & & & & & \\
\hline & Vigna umbellata (Thunb.) Ohwi \& Ohashi 덩굴팥 & & & & & & & & & & & & $\bigcirc$ \\
\hline Lythraceae & Lythrum salicaria L. 털부처꽃 & & & & & & & & & & & & $\bigcirc$ \\
\hline \multirow[t]{4}{*}{ Onagraceae } & Circaea alpina L. 쥐털이슬 & & & & & & & & & & & & $\bigcirc$ \\
\hline & $\begin{array}{l}\text { Circaea lutetiana subsp. quadrisulcata L. (Maxim.) Asch. \& } \\
\text { Magnus 말털이슬 }\end{array}$ & & $\bigcirc$ & & & & & & & & & & \\
\hline & Epilobium glandulosum var. asiaticum H. Hara 줄바늘꽃 & $\bigcirc$ & & & & & & & & & & & \\
\hline & Ludwigia prostrata Roxb. 여뀌바늘 & $\bigcirc$ & & & & & & & & & & & \\
\hline \multirow[t]{2}{*}{ Celastraceae } & $\begin{array}{l}\text { Euonymus alatus for. ciliatodentatus (Franch. \& Sav.) } \\
\text { Hiyama 회잎나무 }\end{array}$ & $\bigcirc$ & & & & & & & & & & & \\
\hline & Euonymus pauciflorus Maxim. 회목나무 & & & & & & & & & & & & $\bigcirc$ \\
\hline Polygalaceae & Polygala tenuifolia Willd. 원지 & & $\bigcirc$ & & & & & & & & & & \\
\hline \multirow[t]{4}{*}{ Aceraceae } & Acer barbinerve Maxim. 청시닥나무 & & & & & & & & & & & $\bigcirc$ & \\
\hline & Acer pictum subsp. mono (Maxim.) Ohashi 고로쇠나무 & $\bigcirc$ & & & & & & & & & & & \\
\hline & Acer pseudo-sieboldianum (Pax) Kom. 당단풍나무 & & & & & & & & & & & & $\bigcirc$ \\
\hline & Acer tataricum subsp. ginnala (Maxim.) Wesm. 신나무 & & & & & & & & & & & & $\bigcirc$ \\
\hline Oxalidaceae & Oxalis obtriangulata Maxim. 큰갱이밥 & & $\bigcirc$ & & & & & & & & & & \\
\hline
\end{tabular}


Table 1. Continued.

\begin{tabular}{|c|c|c|c|c|c|c|c|c|c|c|c|c|c|}
\hline \multirow{2}{*}{ Family } & \multirow{2}{*}{ Taxa } & \multicolumn{12}{|c|}{ Collection date } \\
\hline & & 1 & 2 & 7 & 8 & 9 & 10 & 1213 & 14 & 19 & 20 & 2122 & 223 \\
\hline \multirow[t]{2}{*}{ Geraniaceae } & Geranium knuthii Nakai 큰세 잎쥐손이 & & O & & & & & & & & & & \\
\hline & Geranium krameri Franch. \& Sav. 선이질풀 & $\bigcirc$ & & & & & & & & & & & \\
\hline Balsaminaceae & Impatiens noli-tangere L. 노랑물봉선 & $\bigcirc$ & & & & & & & & & & & $\bigcirc$ \\
\hline Araliaceae & $\begin{array}{l}\text { Eleutherococcus sessiliflorus (Rupr. \& Maxim.) S. Y. Hu } \\
\text { 오갈피나무 }\end{array}$ & & $\bigcirc$ & & & & & & & & & & \\
\hline \multirow[t]{7}{*}{ Apiaceae } & Angelica genuflexa Nutt. ex Torr. \& A. Gray 왜천궁 & $\bigcirc$ & & & & & & & & & & & \\
\hline & Bupleurum longeradiatum Turcz. 개시호 & $\bigcirc$ & & & & & & & & & & & \\
\hline & Cicuta virosa L. 독미나리 & $\bigcirc$ & & & & & & & & & & & \\
\hline & Libanotis coreana (Wolff) Kitagawa 털기름나물 & & & & & & & & & & & & O \\
\hline & Peucedanum terebinthaceum (Fisch.) Fisch. ex DC. 기름나물 & $\bigcirc$ & O & & & & & & & & & & \\
\hline & Pleurospermum camtschaticum Hoffm. 왜우산풀 & & & & & & & & & & & & $\bigcirc$ \\
\hline & Sanicula chinensis Bunge 참반디 & $\bigcirc$ & & & & & & & & & & & \\
\hline \multirow[t]{2}{*}{ Gentianaceae } & Gentiana triflora var. japonica (Kusn.) H. Hara 과남풀 & & & & & & & & & $\bigcirc$ & & & O \\
\hline & Halenia corniculata (L.) Cornaz 닻꽃 & & & & & & & & & & & & 0 \\
\hline Menyanthaceae & Menyanthes trifoliata L. 조름나물 & & & & & & & & & & & $\bigcirc$ & \\
\hline \multirow[t]{2}{*}{ Boraginaceae } & Brachybotrys paridiformis Maxim. ex Oliv. 당개지치 & & & & & & & & & & & & O \\
\hline & Trigonotis radicans (Maxim.) H. Hara 참꽃마리 & & & & & & & & & & & $\bigcirc$ & \\
\hline \multirow[t]{16}{*}{ Lamiaceae } & Agastache rugosa (Fisch. \& Mey.) Kuntze 배초향 & & $\bigcirc$ & & & & & & & & & & O \\
\hline & Amethystea caerulea L. 개차즈기 & & & & & & & & & & & $\bigcirc$ & \\
\hline & Clinopodium chinense var. grandiflora (Maxim.) Kitag. 층층이꽃 & & $\bigcirc$ & & & & & & & & & & \\
\hline & Elsholtzia ciliata (Thunb.) Hyl. 향유 & & & & & & & & & & & & 0 \\
\hline & Galeopsis bifida Boenn. 털향유 & & $\bigcirc$ & & & & & & & & & & \\
\hline & Isodon excisus (Maxim.) Kud 오리방풀 & & & & & & & & & & & & 0 \\
\hline & Leonurus japonicus Houtt. 익모초 & & & & & & & & & & & & 0 \\
\hline & Lycopus uniflorus Michx. 털쉽싸리 & & & & & & & & & & & & $\bigcirc$ \\
\hline & Meehania urticifolia (Miq.) Makino 벌깨덩굴 & & & & & & & & & & & & 0 \\
\hline & Nepeta stewartiana Diels 간장풀 & & & & & & & & & & & $\bigcirc$ & \\
\hline & Phlomis umbrosa Turcz. 속단 & & $\bigcirc$ & & & & & & & & & & \\
\hline & Plectranthus japonicus (Burm.) Koidz. 방아풀 & $\bigcirc$ & & & & & & & & & & & \\
\hline & Prunella vulgaris var. lilacina Nakai 꿀풀 & & & & & & & & $\bigcirc$ & & & & \\
\hline & Scutellaria fauriei H.Lév. \& Vaniot 그늘골무꽃 & & & & & & & & & & & & 0 \\
\hline & Scutellaria indica $\mathrm{L}$. 골무꽃 & & & & & & & & & & & & $\bigcirc$ \\
\hline & Scutellaria pekinensis var transitra (Makino) H. Hara 산골무꽃 & & & & & & & & & & $\bigcirc$ & & \\
\hline Lentibulariaceae & Urticularia intermedia Hayne 개통발 & & & & & & & & & & & $\bigcirc$ & \\
\hline Plantaginaceae & Plantago asiatica $\mathrm{L}$. 질경이 & & & & & & & & & & & & 0 \\
\hline Oleaceae & Fraxinus rhynchophylla Hance 물푸레나무 & $\bigcirc$ & & & & & & & & & & & \\
\hline \multirow[t]{3}{*}{ Scrophulariaceae } & Euphrasia hirtella Jord. 큰산좁쌀풀 & $\bigcirc$ & & & & & & & & & & & \\
\hline & Melampyrum roseum Maxim. 꽃며느리밥풀 & & $\bigcirc$ & & & & & & & & & & O \\
\hline & Pedicularis resupinata $\mathrm{L}$. 송이풀 & & & & & & & & & $\bigcirc$ & & & $\bigcirc$ \\
\hline
\end{tabular}


Table 1. Continued.

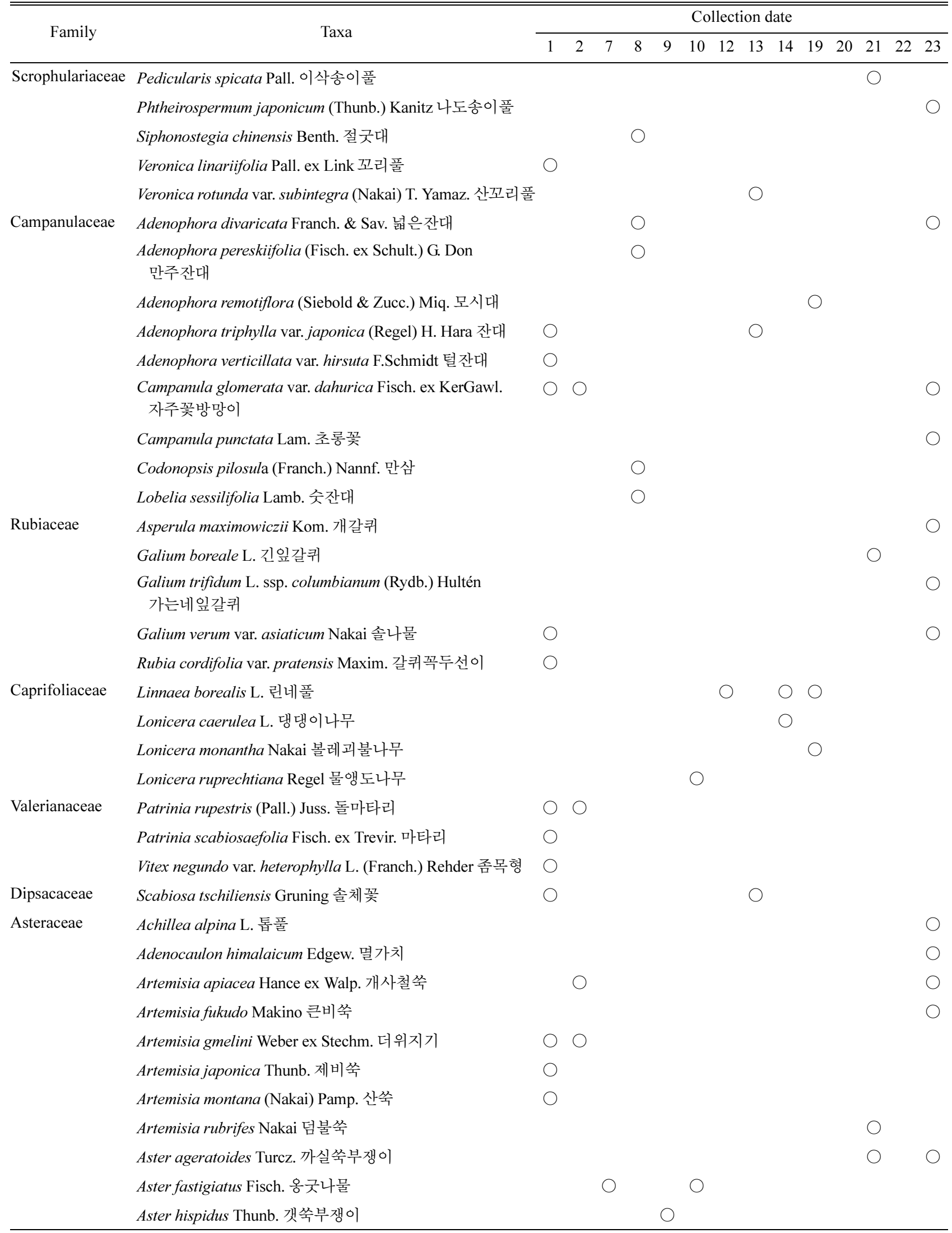


Table 1. Continued.

\begin{tabular}{|c|c|c|c|c|c|c|c|c|c|c|c|c|c|c|c|}
\hline \multirow{2}{*}{ Family } & \multirow{2}{*}{ Taxa } & \multicolumn{14}{|c|}{ Collection date } \\
\hline & & 1 & 2 & 7 & 8 & 9 & 10 & 12 & 13 & 14 & 19 & 20 & 21 & 22 & 23 \\
\hline \multirow[t]{32}{*}{ Asteraceae } & Aster incisus Fisch. 가새쑥부쟁이 & & & & & & & & O & & & & & & \\
\hline & Aster maackii Regel 좀개미취 & & & & & & & & $\bigcirc$ & & & & & & \\
\hline & Aster scaber Thunb. 참취 & O & $\bigcirc$ & & & & & & & & & & & & \\
\hline & Aster tataricus L.f. 개미취 & $\bigcirc$ & & & & & & & $\bigcirc$ & & & & & & $\bigcirc$ \\
\hline & Atractylodes ovata (Thunb.) DC. 삽주 & $\bigcirc$ & & & & & & & & & & & & & \\
\hline & Bidens parviflora Willd. 까치발 & & & & & & & & & & & & & & $\bigcirc$ \\
\hline & $\begin{array}{l}\text { Bidens radiata var. pinnatifida (Turcz. ex DC.) Kitam. } \\
\text { 구와가막사리 }\end{array}$ & & & & & & & & & & & & & & 0 \\
\hline & Breea setosa (Wild.) Kitam. 큰조뱅이 & & & & & & & & & & & & & & $\bigcirc$ \\
\hline & Cacalia auriculata (DC.) J.R. Grant 귀박쥐나물 & & & & & & & & & & & & $\bigcirc$ & & \\
\hline & Callistephus chinensis (L.) Nees 과꽃 & $\bigcirc$ & & & & & & & & & & & $\bigcirc$ & & \\
\hline & Carthamus tinctorius L. 잇꽃 & & & & & & & & & & & & & & $\bigcirc$ \\
\hline & Chrysanthemum alpinum L. 고산구절초 & & & & & & & & & $\bigcirc$ & & & & & \\
\hline & Chrysosplenium sp. & & & & & & & & & & & & $\bigcirc$ & & \\
\hline & Cirsium vlassovianum Fisch. ex DC. 흰잎엉겅퀴 & & & & & & & & $\bigcirc$ & & & & $\bigcirc$ & & \\
\hline & Dendranthema naktongense (Nakai) Tzvelev 구절초 & & & & & & & & & & & & & $\bigcirc$ & \\
\hline & Hieracium umbellatum L. 조밥나물 & & $\bigcirc$ & & & & & & & & & & & & $\bigcirc$ \\
\hline & Inula britannica var. linariifolia (Turcz.) Regel 가는금불초 & & & & & & & & & & & & & & $\bigcirc$ \\
\hline & Kalimeris integrifolia Turcz. ex DC. 가는쑥부쟁이 & & & & & & & & & & & & & & $\bigcirc$ \\
\hline & Lactuca raddeana Maxim. 산씀바귀 & & & & & & & & & & & & & & $\bigcirc$ \\
\hline & Lactuca sibirica (L.) Benth. ex Maxim. 자주방가지똥 & & & & O & & & & & & & & & & \\
\hline & Picris hieracioides var. koreana Kitam. 쇠서나물 & & & & & & & & & & & & & & $\bigcirc$ \\
\hline & Saussurea grandifolia Maxim. 서덜취 & & $\bigcirc$ & & & & & & & & & & & & \\
\hline & Saussurea manshurica Kom. 덤불취 & & & & $\bigcirc$ & & & & & & & & & & \\
\hline & Saussurea neoserrata Nakai 산골취 & & & & $\bigcirc$ & & & & & & & & & & \\
\hline & Saussurea ussuriensis Maxim. 구와취 & & & $\bigcirc$ & & & & & & & & & & & \\
\hline & Scorzonera albicaulis Bunge 쇠채 & & O & & & & & & & & & & & & \\
\hline & Senecio cannabifolius Less. 삼잎 방망이 & & & & & & & & & $\bigcirc$ & & & & & \\
\hline & Senecio nemorensis L. 금방망이 & & & & & & & & & & & & & & 0 \\
\hline & Sigesbeckia pubescens Makino 털진득찰 & & $\bigcirc$ & & & & & & & & & & & & $\bigcirc$ \\
\hline & Solidago virg-aurea subsp. asiatica Kitam. ex Hara 미역취 & & & & & & & & & & & & & & $\bigcirc$ \\
\hline & Sonchus brachyotus DC. 사데풀 & & & & O & & & & & & & & & & \\
\hline & Taraxacum platycarpum Dahlst. 민들레 & & & & & & & & & & & & & & O \\
\hline \multirow[t]{2}{*}{ Juncaceae } & Juncus laschenaultii Gay ex Laharpe 참비녀골풀 & & & & & & & & $\bigcirc$ & & & & & & \\
\hline & Luzula wahlenbergii Rupr. 좀뀡의밥 & & & & & & & & & & & & & & $\bigcirc$ \\
\hline \multirow[t]{2}{*}{ Cyperaceae } & Carex phacota Spreng 쥐방울사초 & & & & & & & & & & & & & & $\bigcirc$ \\
\hline & Carex siderosticta Hance 대사초 & & & & & & & & & & & & & & $\bigcirc$ \\
\hline \multirow[t]{4}{*}{ Poaceae } & Agrostis clavata var. clavata Trin. 산겨이삭 & & & & & & & & & & & & & & 0 \\
\hline & Arundinella hirta (Thunb.) Koidz. 새 & $\bigcirc$ & & & & & & & & & & & & & \\
\hline & Festuca takedana Ohwi 개묵새 & $\bigcirc$ & & & & & & & & & & & & & \\
\hline & Milium effusum L. 나도겨이삭 & & & & & & & & & & $\bigcirc$ & & & & \\
\hline
\end{tabular}


Table 1. Continued.

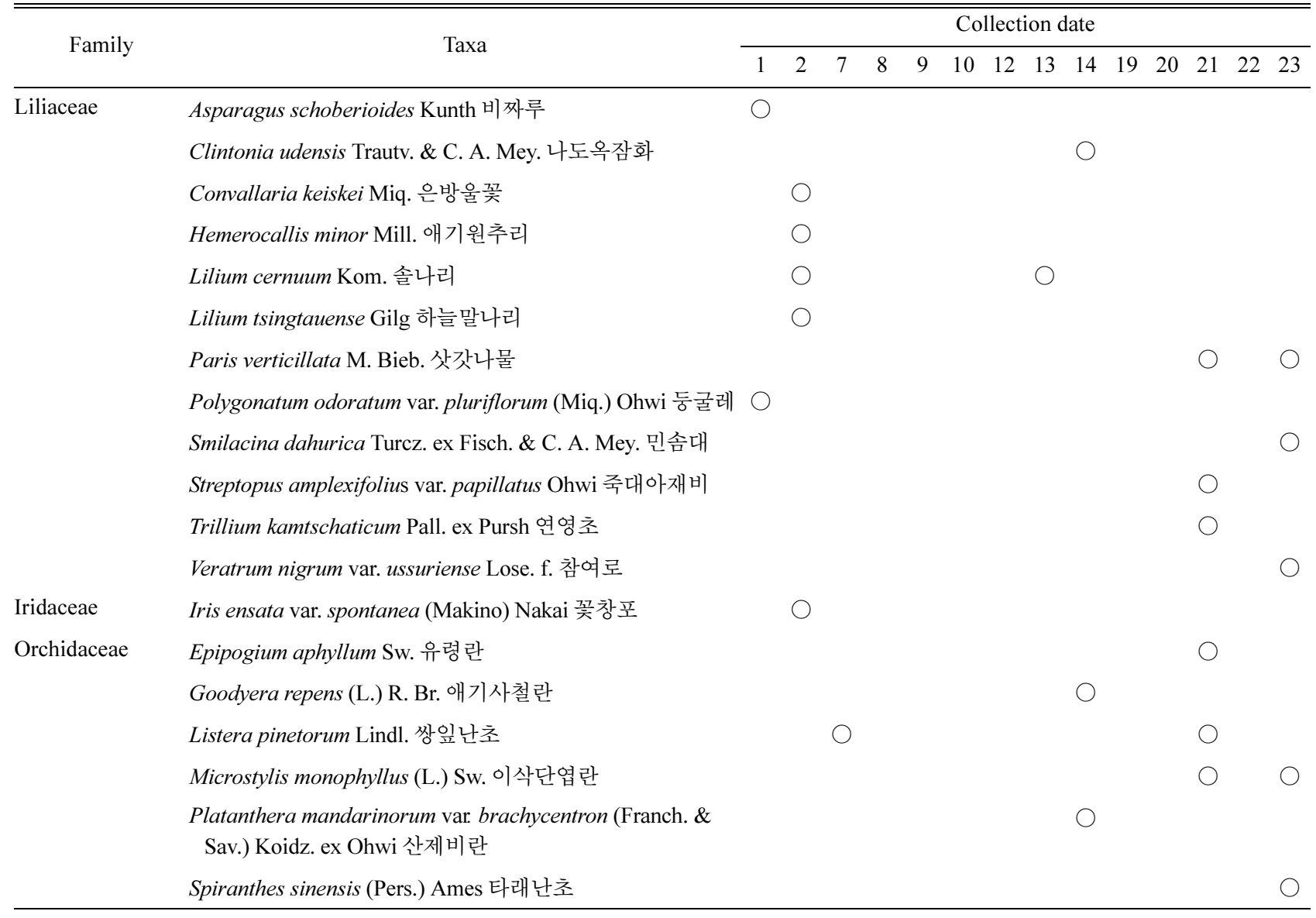

